\title{
Description of Desulfotomaculum sp. Groll as Desulfotomaculum gibsoniae sp. nov.
}

\author{
Jan Kuever, ${ }^{1}$ Fred A. Rainey ${ }^{2}$ and Hans Hippe ${ }^{3}$ \\ Author for correspondence: Jan Kuever. Tel: +49 4212028 734. Fax : +49 4212028580. \\ e-mail: jkuever@mpi-bremen.de
}

\footnotetext{
1 Max-Planck-Institute for Marine Microbiology, Celsiusstraße 1, D-28359 Bremen, Germany

2 Department of Biological Sciences, 508 Life Sciences Building, Louisiana State University, Baton Rouge, LA 70803, USA

${ }^{3}$ DSMZ - Deutsche Sammlung von Mikroorganismen und Zellkulturen $\mathrm{GmbH}$, D-38124 Braunschweig, Germany
}

\begin{abstract}
Strain Groll', isolated from fresh water, is a mesophilic, spore-forming, sulfatereducing bacterium that uses a large variety of substrates as electron donors ranging from simple organic compounds to long-chain fatty acids and several aromatic compounds. Sulfate, thiosulfate and sulfite are used as electron acceptors. Homoacetogenic growth occurs under sulfate-free conditions. Substrate oxidation is usually complete, leading to $\mathrm{CO}_{2}$, but acetate or other fatty acids can accumulate at high substrate concentrations. The $\mathrm{G}+\mathrm{C}$ content of the DNA is $54.8 \mathrm{~mol} \%$. Strain Groll' was found to be phenotypically and phylogenetically different from known members of the genus Desulfotomaculum. 16S rRNA gene sequence analyses show that this organism falls within the radiation of the genus Desulfotomaculum cluster and has $<96 \%$ sequence similarity to previously described species. The name Desulfotomaculum gibsoniae sp. nov. is proposed for this strain; the type strain is Groll' (=DSM 7213').
\end{abstract}

Keywords: Desulfotomaculum, sulfate-reducing bacterium, anaerobic degradation of aromatic compounds

\section{INTRODUCTION}

All spore-forming, dissimilatory sulfate-reducing bacteria were originally classified in the genus Desulfotomaculum (Campbell \& Singleton, 1986; Widdel, 1992). Phylogenetically, they are members of the Clostridium-Bacillus subphylum of the Gram-positive bacteria (Stackebrandt et al., 1997) and comprise mesophilic and moderately thermophilic species that show either complete or incomplete oxidation of organic substrates with concomitant reduction of sulfate and other sulfur oxyanions to sulfide (Widdel \& Pfennig, 1977, 1981; Nazina et al., 1989; Min \& Zinder, 1990). Recent detailed phylogenetic analysis of the majority of the described species of the genus Desulfotomaculum and additional strains has demonstrated that the genus is not monophyletic but rather contains two different branches (Stackebrandt et al., 1997). The majority of the species and isolates form one phylogenetically coherent cluster, whereas the strains of Desulfotomaculum orientis represent an additional lineage closely related to the genus Desulfitobacterium. These strains have therefore been described as a novel genus, Desulfosporosinus. While the

The EMBL accession number for the 16S rRNA gene sequence of strain Groll' is $Y 11576$. taxonomic status of the Desulfotomaculum guttoideum is unclear, this strain shows high sequence similarity to a previously described Clostridium species (Stackebrandt et al., 1997). Since the phylogenetic survey of the genus was presented (Stackebrandt et al., 1997), four additional species have been added to the genus: Desulfotomaculum putei (Liu et al., 1997), Desulfotomaculum luciae (Liu et al., 1997), 'Desulfotomaculum auripigmentum' (Newman et al., 1997) and Desulfotomaculum halophilum (Tardy-Jacquenod et al., 1998). With the exception of 'Desulfotomaculum auripigmentum', which shows highest sequence similarity to the genus Desulfosporosinus, these newly described species add further phylogenetic diversity to the genus $D e-$ sulfotomaculum.

Members of these genera seem to be well adapted to changing redox conditions, facilitated by the formation of spores. They can be found in marine and freshwater ecosystems, including thermally heated groundwaters and oilfield brines, and were even isolated from corroded aluminium alloys (Widdel \& Pfennig, 1981; Cord-Ruwisch \& Garcia, 1985; Daumas et al., 1988; Nazina et al., 1989; Hagenauer et al., 1997; Hippe et al., 1997; Tardy-Jacquenod et al., 1998). The increase of new descriptions of sporeforming, sulfate-reducing bacteria in the last few years demonstrates the abundance and ecological import- 
ance of this specialized group (Min \& Zinder, 1990; Tasaki et al., 1991; Kuever et al., 1993; Vainshtein et al., 1995; Liu et al., 1997; Love et al., 1993; Nilsen et al., 1996). The broad substrate spectrum of organisms of this group, ranging from simple organic compounds to long-chain fatty acids and several different aromatic compounds, implies that they must play a major role in the breakdown of organic matter under anoxic conditions.

As reported by several authors, some spore-forming, sulfate-reducing bacteria show homoacetogenic growth under sulfate-free conditions, which gives them the ability to survive even under sulfate-limiting conditions, as found typically in freshwater sediments or aquifers (Klemps et al., 1985; Tasaki et al., 1991; Kuever et al., 1993; Hanselmann et al., 1995). Here, we describe an additional new species of the genus Desulfotomaculum isolated from a freshwater mud sample.

\section{METHODS}

Source of organism. Strain Groll ${ }^{\mathrm{T}}$ was isolated from an enrichment culture inoculated with a black-mud sample from a small freshwater ditch in Bremen, northern Germany. Benzoate was used as the only carbon source and electron donor. A pure culture was obtained by repeated application of the agar dilution method. The strain was deposited with DSMZ under the accession number DSM $7213^{\mathrm{T}}$

Culture methods and media. The organism was cultivated as described previously (Kuever et al., 1993).

Chemical and biochemical characterization. The presence of desulfoviridin was tested as described by Postgate (1956). The whole-cell fatty acid composition was determined by means of capillary GC using cell material of strains grown under identical conditions (Vainshtein et al., 1992).

Phylogenetic analyses of $16 \mathrm{~S}$ rRNA gene sequences. $16 \mathrm{~S}$ rRNA gene sequences available from the public databases were aligned using the ae2 editor (Maidak et al., 1996). The strain designations and nucleotide sequence accession numbers are as follows: Desulfotomaculum acetoxidans DSM 771 ${ }^{\mathrm{T}}$, Y11566; Desulfotomaculum aeronauticum DSM $10349^{\mathrm{T}}$, X98407; Desulfotomaculum australicum ACM 3917 ${ }^{\mathrm{T}}$, M96665; Desulfotomaculum geothermicum DSM $3669^{\mathrm{T}}$, Y11567; Desulfotomaculum halophilum DSM 11559, U88891; Desulfotomaculum kuznetsovii DSM 6115', Y11569; Desulfotomaculum luciae SMCC W644,', AF069293; Desulfotomaculum nigrificans NCIMB 8395', X62176; Desulfotomaculum putei SMCC W459 ${ }^{\mathrm{T}}$, AF053929; Desulfotomaculum ruminis DSM 2154, Y11572; Desulfotomaculum sapomandens DSM 3223 ${ }^{\mathrm{T}}$, AF168365; Desulfotomaculum thermoacetoxidans DSM 5813 ${ }^{\mathrm{T}}$, Y11573; Desulfotomaculum thermobenzoicum DSM $6193^{\mathrm{T}}$, Y11574; Desulfotomaculum thermocisternum DSM $10259^{\mathrm{T}}$, U33455; Desulfotomaculum thermosapovorans DSM 6562 ${ }^{\mathrm{T}}, \mathrm{Y} 11575$; Desulfotomaculum sp. DSM 7213, Y11576; Desulfotomaculum sp. DSM 7440, Y11579; Desulfotomaculum sp. DSM 7474, Y11577; Desulfotomaculum sp. DSM 7475, Y11580; Desulfotomaculum sp. DSM 7476, Y11578; and Desulfosporosinus orientis DSM $765^{\mathrm{T}}$, Y11570. After removal of the $5^{\prime}$ end of each sequence because of variations in sequence length and alignment uncertainty, the alignment used in the phylogenetic analyses comprised 1100 nucleotide positions between positions 109 and 1387 (Escherichia coli numbering). The programs of the PHYLIP package, including DNADIST and NEIGHBOUR, were used for the phylogenetic analyses (Felsenstein, 1993). The tree topology was reanalysed using 500 bootstrapped data sets and the programs SEQBOOT, DNADIST and CONSENSE of the PHYLIP package (Felsenstein, 1993).

\section{RESULTS}

\section{Enrichment and isolation}

Media containing benzoate as the only carbon source were inoculated with black, anaerobic mud from a freshwater ditch. After 2 weeks incubation, formation of sulfide and growth of large spore-forming rods showing slow motility were observed. After several transfers into liquid medium, a pure culture was obtained by repeated agar dilution series. Additional experiments showed that benzyl alcohol was a very selective substrate for enrichment of Desulfotomaculum strains similar to strain Groll ${ }^{\mathrm{T}}$. Desulfococcus multivorans strains, which are also common benzoateutilizing, sulfate-reducing bacteria in freshwater habitats, were unable to use this aromatic compound for growth.

\section{Cell morphology}

Vegetative cells of strain Groll ${ }^{\mathrm{T}}$ grown with butyrate and sulfate were straight or slightly curved rods measuring 1.0-2.5 $\times 4-7 \mu \mathrm{m}$ with pointed ends. The spores were spherical and located in the centre of the cells, causing swelling. Vegetative cells were actively motile, but motility was lost during cultivation. The arrangement of flagella therefore could not be determined.

\section{DNA G + C content}

The determination of the DNA base ratio yielded a $\mathrm{G}+\mathrm{C}$ content of $54.8 \mathrm{~mol} \%$ (Kuever et al., 1993).

\section{Physiological properties}

Desulfoviridin was absent in strain Groll ${ }^{\mathrm{T}}$. The optimum growth temperature of strain $\mathrm{Groll}^{\mathrm{T}}$ was determined to be $35-37^{\circ} \mathrm{C}$. No growth was observed at temperatures greater than $40{ }^{\circ} \mathrm{C}$ or lower than $20^{\circ} \mathrm{C}$. The $\mathrm{pH}$ range for growth was between 6.0 and 8.0 with an optimum at $\mathrm{pH} 7 \cdot 0-7 \cdot 4$. Vitamins were not essential for growth. After transfer to fresh medium, the isolate required sodium dithionite $(100 \mu \mathrm{M})$ as additional reductant to initiate growth.

Strain Groll ${ }^{\mathrm{T}}$ used the following compounds as electron donors and carbon sources in the presence of $10 \mathrm{mM}$ sulfate (concentrations of added substrates, in $\mathrm{mM}$ except where indicated, are given in parentheses) (Kuever et al., 1993; Londrey et al., 1997): $\mathrm{H}_{2}$ plus $\mathrm{CO}_{2}(80: 20 ; \mathrm{v} / \mathrm{v})$, formate (20), propionate (10), butyrate (5), valerate (5), caproate (3), caprylate (2), caprinate (2), isobutyrate (5), 2-methylbutyrate (5), 3methylbutyrate (5), crotonate (8), ethanol (10), 1propanol (5), 1-butanol (5), 1-hexanol (5), 1-octanol 
Table 1. Stoichiometric measurements with strain Groll' after growth on butanol as electron donor and carbon source and sulfate as electron acceptor

\begin{tabular}{|c|c|c|c|c|c|c|}
\hline $\begin{array}{l}\text { Substrate added } \\
\quad(\mathrm{mM})\end{array}$ & $\begin{array}{l}\text { Acetate (butyrate) } \\
\text { excreted (mM) }\end{array}$ & $\begin{array}{l}\text { Cell dry weight } \\
\text { formed }\left(\mathrm{mg} \mathrm{ml}^{-1}\right)\end{array}$ & $\begin{array}{c}\text { Substrate } \\
\text { consumed for } \\
\text { cell material* } \\
(\mathbf{m M})\end{array}$ & $\begin{array}{c}\text { Substrate } \\
\text { oxidized by } \\
\text { sulfate reduction } \\
(\mathbf{m M})\end{array}$ & $\begin{array}{l}\text { Sulfide produced } \\
\text { per mol substrate } \\
\text { oxidized (mol) }\end{array}$ & $\begin{array}{c}\text { Growth yield } \\
\text { (g dry weight } \\
\text { mol }^{-1} \text { substrate } \\
\text { oxidized) }\end{array}$ \\
\hline $0 \cdot 5$ & 0 & $13 \cdot 8$ & $0 \cdot 10$ & $0 \cdot 42$ & $3 \cdot 33$ & $32 \cdot 9$ \\
\hline 0.9 & 0 & $21 \cdot 0$ & $0 \cdot 14$ & 0.76 & $3 \cdot 16$ & $27 \cdot 6$ \\
\hline $1 \cdot 3$ & $1 \cdot 1$ & $23 \cdot 9$ & $0 \cdot 16$ & $1 \cdot 14$ & $2 \cdot 02$ & $23 \cdot 0$ \\
\hline 1.6 & $2 \cdot 0$ & $28 \cdot 3$ & $0 \cdot 20$ & $1 \cdot 40$ & $1 \cdot 86$ & $20 \cdot 2$ \\
\hline $2 \cdot 1$ & $4 \cdot 3$ & $29 \cdot 2$ & $0 \cdot 20$ & 1.90 & $1 \cdot 26$ & $15 \cdot 4$ \\
\hline 3.9 & $7 \cdot 9$ & $47 \cdot 2$ & $0 \cdot 32$ & $3 \cdot 58$ & $1 \cdot 17$ & $13 \cdot 2$ \\
\hline $8 \cdot 5$ & $10 \cdot 2(2 \cdot 9)$ & $68 \cdot 5$ & $0 \cdot 47$ & 8.03 & $0 \cdot 81$ & $8 \cdot 5$ \\
\hline $13 \cdot 5$ & $14 \cdot 6(6 \cdot 2)$ & $81 \cdot 8$ & 0.56 & $12 \cdot 94$ & $0 \cdot 80$ & $6 \cdot 3$ \\
\hline $19 \cdot 0$ & $16 \cdot 6(9 \cdot 6)$ & $93 \cdot 4$ & $0 \cdot 64$ & $18 \cdot 36$ & 0.73 & $5 \cdot 1$ \\
\hline
\end{tabular}

* Substrate consumed for cell material was calculated by the following equation: $17 \mathrm{C}_{4} \mathrm{H}_{10} \mathrm{O}+28 \mathrm{HCO}_{3}^{-} \rightarrow 24\left(\mathrm{C}_{4} \mathrm{H}_{7} \mathrm{O}_{3}\right)$ $+28 \mathrm{OH}^{-}+\mathrm{H}_{2} \mathrm{O}$; thus $0.0069 \mathrm{mmol} 1$-butanol is required for $1.0 \mathrm{mg}$ cell dry weight.

(2), 2-methyl-1-propanol (5), 1,3-propanediol (5), pyruvate (10), malate (5), fumarate (5), succinate (5), maleate (5), benzoate (2), 3-hydroxybenzoate (2), 4hydroxybenzoate (2), 2-aminobenzoate (2), 2-fluorobenzoate $(0.5), 4$-fluorobenzoate $(0.5)$, 3-methylbenzoate $(0.5)$, phenol (2), catechol (2), 4-methylcatechol $(0 \cdot 5), m$-cresol (1), p-cresol (1), 4-hydroxybenzyl alcohol (1), 3-hydroxybenzyl alcohol (0.5), benzyl alcohol (2), 4-hydroxybenzaldehyde (1), 3hydroxybenzaldehyde $(0 \cdot 5)$, benzaldehyde $(2), 3,4$ dihydroxybenzaldehyde (1), 3,4-dihydroxybenzoate (2), phenylacetate (2), phenylpropionate (2), phenylbutyrate (2), vanillyl alcohol (1), vanillin (2), vanillate (2), anisol (1), cinnamate (2) and ferulate (2).

Substrates tested but not utilized were: fructose (2), glucose (2), xylose (2), maltose (2), galactose (2), cellobiose (2), methanol (20), methanol (20) plus acetate (2), 2-propanol (5), 2-butanol (5), glycerol (5), cyclohexanol (1), cyclohexanone (1), cyclohexane carboxylate (2), acetate (10), undecanate (1), laurinate $(0 \cdot 5)$, myristate $(1)$, palmitate $(1 \cdot 5)$, stearate $(1 \cdot 5)$, cysteine (2), glycine (5), aspartate (2), lactate (10), citrate (5), tartrate (5), oxalate (10), malonate (10), adipate (3), 2-hydroxybenzoate (2), phthalate (2), gallate (2), 3-methoxybenzoate (2), 4-methoxybenzoate (2), 2,4-dihydroxybenzoate (2), 2,5-dihydroxybenzoate (2), 3,5-dihydroxybenzoate (2), 4aminobenzoate (2), resorcinol (1), hydroquinone (1), pyrogallol (1), phloroglucinol (1), hydroxyhydroquinone (1), 2-, 3- and 4-aminophenol (each at 0.5), 2,4-diaminophenol $(0 \cdot 5)$, anilin $(0 \cdot 5)$, 4-nitrophenol $(0.5)$, toluene $(0.5)$, 4-hydroxybiphenyl $(0.5)$, 2hydroxybiphenyl $(0 \cdot 5)$, nicotinate (2) and phenylalanine (2).

Sulfate, sulfite and thiosulfate were used as electron acceptors. Sulfur and nitrate did not serve as electron acceptors. Pyruvate, crotonate, formate, hydrogen plus carbon dioxide $(80: 20, \mathrm{v} / \mathrm{v})$ and methoxylated aromatic compounds allowed slow growth by homoacetogenesis when yeast extract was supplied as a supplement.

Fastest growth occurred with butyrate and 1-butanol, with a minimum doubling time of 12-14 h. Increased substrate concentrations resulted in the production of acetate or other fatty acids, depending on the substrate (Table 1). Furthermore, high concentrations of 1butanol led to butyrate and acetate production. Some aromatic compounds were transformed by $O$ demethylation or decarboxylation prior to complete oxidation. Sodium chloride was not required for growth, although the isolate tolerated salt concentrations up to $35 \mathrm{~g}^{-1}$.

\section{Cellular fatty acid composition}

The fatty acid compositions of strain $\mathrm{Groll}^{\mathrm{T}}$ and its closest phylogenetically related species are listed in Table 2. Significant amounts of unidentified fatty acids (ECL 11.86, ECL 13.05, ECL 13.26) were found in strain Groll ${ }^{\mathrm{T}}(19 \cdot 2 \%)$, Desulfotomaculum geothermicum $(13 \%)$ and Desulfotomaculum thermosapovorans $(8.5 \%)$.

\section{Phylogenetic analyses}

The phylogenetic position of strain Groll ${ }^{\mathrm{T}}$ has been shown previously and its novel species status within the genus Desulfotomaculum has been demonstrated (Stackebrandt et al., 1997). Comparative phylogenetic analyses, which included four additional recently described Desulfotomaculum species, showed strain Groll $^{\mathrm{T}}$ to represent a distinct lineage within subcluster $\mathrm{Ib}$ and not to be closely related to any of these newly described taxa (Fig. 1). The 16S rRNA gene sequence of strain Groll $^{\mathrm{T}}$ shared $94 \cdot 0-95 \cdot 5 \%$ similarity with the 16S rRNA gene sequences of the three other Desulfotomaculum species that comprise cluster Ib, namely 
Table 2. Fatty acid composition of Desulfotomaculum gibsoniae and phylogenetically related Desulfotomaculum species

Percentages of total fatty acids are shown. Fatty acids present in all strains at less than $1 \%$ are not listed. ECL, Equivalent chain-length; dma, dimethyl acetal (equivalent to aldehyde); c, cis; i, iso; ai, anteiso; ald, aldehyde; cyc, cyclopropane.

\begin{tabular}{|c|c|c|c|c|c|}
\hline ECL & Fatty acid methyl ester & $\begin{array}{l}\text { D. gibsoniae } \\
\text { strain Groll }{ }^{\mathrm{T}}\end{array}$ & $\begin{array}{l}\text { D. geothermicum } \\
\text { DSM } 3669^{\mathrm{T}}\end{array}$ & $\begin{array}{l}\text { D. thermosapovorans } \\
\text { DSM } 6562^{\mathrm{T}}\end{array}$ & $\begin{array}{l}\text { D. sapomandens } \\
\text { DSM } 3223^{\mathrm{T}}\end{array}$ \\
\hline $11 \cdot 00$ & $11: 0$ & 1.9 & 0.9 & 0.5 & - \\
\hline $13 \cdot 60$ & i14:0 & $1 \cdot 6$ & - & $1 \cdot 4$ & - \\
\hline $14 \cdot 00$ & $14: 0$ & $2 \cdot 6$ & 7.9 & $2 \cdot 1$ & $1 \cdot 0$ \\
\hline $14 \cdot 41$ & i1 $15: 1$ & - & 6.5 & $1 \cdot 0$ & 0.5 \\
\hline $14 \cdot 62$ & i1 $5: 0$ & $5 \cdot 4$ & $32 \cdot 8$ & $23 \cdot 1$ & $2 \cdot 0$ \\
\hline $14 \cdot 71$ & ai15:0 & - & $1 \cdot 4$ & - & - \\
\hline $14 \cdot 79$ & $15: 1 c 7$ & $2 \cdot 7$ & - & - & $0 \cdot 6$ \\
\hline $14 \cdot 95$ & $16: 0$ ald & $2 \cdot 2$ & - & 0.6 & - \\
\hline $15 \cdot 00$ & $15: 0$ & - & 1.5 & $1 \cdot 4$ & 0.8 \\
\hline $15 \cdot 11$ & i15:0 dma & - & $2 \cdot 2$ & $1 \cdot 2$ & - \\
\hline $15 \cdot 46$ & $15: 0 \mathrm{dma}$ & 1.7 & - & - & $5 \cdot 4$ \\
\hline $15 \cdot 63$ & i16:0 & - & 1.6 & $2 \cdot 6$ & $3 \cdot 5$ \\
\hline $15 \cdot 77$ & $16: 1 c 7$ & $3 \cdot 3$ & - & 0.7 & 6.5 \\
\hline $15 \cdot 82$ & $16: 1 c 9$ & $15 \cdot 1$ & $4 \cdot 0$ & $3 \cdot 6$ & $21 \cdot 3$ \\
\hline $15 \cdot 91$ & $16: 1 c 11$ & $3 \cdot 6$ & - & - & 6.2 \\
\hline $16 \cdot 00$ & $16: 0$ & $9 \cdot 6$ & $10 \cdot 1$ & $15 \cdot 7$ & $10 \cdot 2$ \\
\hline $16 \cdot 29$ & $16: 1 c 9 \mathrm{dma}$ & $4 \cdot 6$ & - & $1 \cdot 1$ & $4 \cdot 2$ \\
\hline $16 \cdot 42$ & $\mathrm{i} 17: 1 c 7$ & $4 \cdot 8$ & $7 \cdot 2$ & $5 \cdot 0$ & $4 \cdot 6$ \\
\hline $16 \cdot 48$ & $16: 0 \mathrm{dma}$ & $6 \cdot 7$ & $5 \cdot 0$ & $3 \cdot 9$ & $1 \cdot 8$ \\
\hline $16 \cdot 63$ & i17:0 & - & $2 \cdot 4$ & 8.9 & 0.7 \\
\hline $16 \cdot 80$ & $17: 1 c 9$ & - & - & - & $1 \cdot 0$ \\
\hline 16.87 & $17: 1 c 11$ & - & - & - & 1.9 \\
\hline 16.89 & $17: 0 \mathrm{cyc}$ & $2 \cdot 7$ & $3 \cdot 7$ & $5 \cdot 8$ & - \\
\hline 17.00 & $17: 0$ & - & - & $1 \cdot 4$ & 0.5 \\
\hline $17 \cdot 10$ & $\mathrm{i} 17: 0 \mathrm{dma}$ & - & - & $7 \cdot 5$ & - \\
\hline $17 \cdot 47$ & $17: 0 \mathrm{dma}$ & - & - & - & 1.7 \\
\hline 17.77 & $18: 1 c 9$ & $2 \cdot 0$ & - & - & $3 \cdot 4$ \\
\hline 17.82 & $18: 1 \mathrm{c} 11$ & $3 \cdot 3$ & - & 0.9 & $6 \cdot 9$ \\
\hline 17.92 & $18: 1 c 13$ & $2 \cdot 8$ & - & - & $2 \cdot 5$ \\
\hline $18 \cdot 00$ & $18: 0$ & $5 \cdot 6$ & - & $3 \cdot 2$ & $4 \cdot 4$ \\
\hline $18 \cdot 29$ & $18: 1 c 11 \mathrm{dma}$ & $1 \cdot 5$ & - & - & $1 \cdot 4$ \\
\hline \multicolumn{2}{|c|}{ Total unidentified } & $19 \cdot 2$ & $13 \cdot 0$ & $8 \cdot 5$ & $1 \cdot 7$ \\
\hline \multicolumn{2}{|c|}{ Saturated, even-numbered } & $26 \cdot 7$ & $23 \cdot 0$ & $25 \cdot 5$ & $7 \cdot 2$ \\
\hline \multicolumn{2}{|c|}{ Saturated, odd-numbered } & $3 \cdot 6$ & $2 \cdot 4$ & $3 \cdot 3$ & $8 \cdot 4$ \\
\hline \multicolumn{2}{|c|}{ Branched, iso } & 11.8 & $52 \cdot 7$ & $50 \cdot 7$ & $11 \cdot 3$ \\
\hline \multicolumn{2}{|c|}{ Branched, anteiso } & $0 \cdot 0$ & $1 \cdot 4$ & $0 \cdot 0$ & 0.0 \\
\hline \multicolumn{2}{|c|}{ Unsaturated } & $43 \cdot 7$ & 17.7 & $12 \cdot 3$ & $61 \cdot 8$ \\
\hline \multicolumn{2}{|c|}{ Dimethyl acetals } & $14 \cdot 5$ & $7 \cdot 2$ & $13 \cdot 7$ & $15 \cdot 4$ \\
\hline \multicolumn{2}{|c|}{ Cyclopropane fatty acids } & $2 \cdot 7$ & $3 \cdot 7$ & $5 \cdot 8$ & $2 \cdot 8$ \\
\hline
\end{tabular}

Desulfotomaculum geothermicum $(94 \cdot 0 \%)$, Desulfotomaculum thermosapovorans $(94.9 \%)$ and Desulfotomaculum sapomandens $(95 \cdot 5 \%)$. The recently described Desulfotomaculum species all fell within the different clusters defined by Stackebrandt et al. (1997). Desulfotomaculum putei grouped with Desulfotomaculum sp. strain DSM 7740 in subcluster Ia (Fig. 1), the $16 \mathrm{~S}$ rRNA gene sequences of these two taxa sharing $98.0 \%$ similarity and the sequence of Desulfotom- aculum putei having all of the signature nucleotides designated for this subcluster (Stackebrandt et al., 1997). The 16S rRNA gene sequence of Desulfotomaculum luciae, with a few exceptions, contains most of the signature nucleotides designated for subcluster Ic. These exceptions include A-U (compared with G-U) at positions 293-304 and G--U (compared with $\mathrm{G}-\mathrm{C})$ at positions $662-743$. The thermophilic species Desulfotomaculum luciae showed highest 16S rRNA 


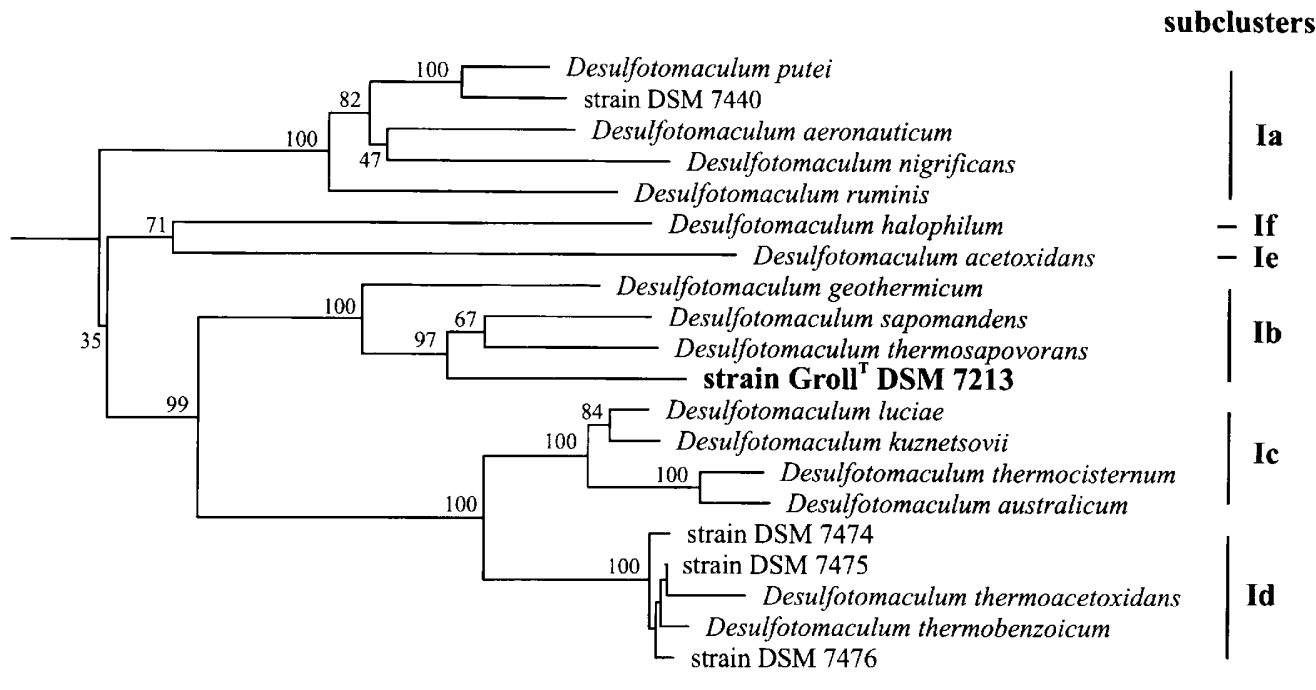

$10 \%$

Fig. 1. Phylogenetic dendrogram based on $16 \mathrm{~S}$ rRNA gene sequence comparisons of all validly described species and additional strains of the genus Desulfotomaculum. The position of the root was determined by the inclusion of Desulfosporosinus orientis as the outgroup organism. The neighbour-joining tree was reconstructed from distance matrices. Bootstrap values from the analyses of 500 data sets (expressed as percentages) are shown at the branching points. The scale bar represents 10 nucleotide substitutions per 100 nucleotides. The phylogenetic subclusters of cluster 1 as previously defined (Stackebrandt et al., 1997) and the newly defined subcluster If are indicated.

gene sequence similarity to another thermophile, Desulfotomaculum kuznetsovii (99.4\%). The species status of Desulfotomaculum luciae requires further clarification at the DNA-DNA homology level considering this high $16 \mathrm{~S}$ rRNA gene sequence similarity, although there are significant phenotypic differences between these two species. The recently described halophilic species Desulfotomaculum halophilum represents a distinct lineage within cluster I, with $16 \mathrm{~S}$ rRNA gene sequence similarity values to the other members of cluster $I$ in the range $86.8-88.6 \%$. Although it clustered with Desulfotomaculum acetoxidans (Fig. 1), the bootstrap value of $71 \%$ indicates a less than significant branching order. Comparison of the nucleotide signatures defined for subcluster Ie (Stackebrandt et al., 1997) (of which Desulfotomaculum acetoxidans is the only member) with the $16 \mathrm{~S}$ rRNA gene sequence of Desulfotomaculum halophilum showed that only 20 of the 33 signatures defined are present. This finding, in combination with the low bootstrap and sequence similarity values, indicates that Desulfotomaculum halophilum should be assigned to an additional single species cluster or lineage, designated cluster If. The fourth recently described Desulfotomaculum species, 'Desulfotomaculum auripigmentum', clustered with the Desulfosporosinus orientis strains, showing 97.5 and $98.3 \%$ 16S rRNA gene sequence similarity, respectively, to strains DSM $765^{\mathrm{T}}$ and DSM 8344 (data not shown). Further phenotypic and physiological comparisons are required before 'Desulfotomaculum auripigmentum' can be reclassified to the genus Desulfosporosinus.

\section{DISCUSSION}

A general feature of members of the genus Desulfotomaculum seems to be that increasing substrate concentrations can lead to incomplete oxidation of the organic substrates, although complete oxidation was shown for low substrate concentrations (Daumas et al., 1988; Fardeau et al., 1995). At low substrate concentrations, strain Groll ${ }^{\mathrm{T}}$ showed complete oxidation of 1-butanol to $\mathrm{CO}_{2}$ (see equation 1). Above a concentration of $1 \mathrm{mM}$, a stoichiometric release of acetate from butanol was observed (see equation 2), which might be a result of a CoA transferase reaction, as proposed for Desulfobacterium autotrophicum (Schauder et al., 1986). In this reaction, one of the two acetyl CoA molecules resulting from the $\beta$-oxidation of butyrate is used for activation of the next butyrate to butyryl-CoA. Butyrate should be an intermediate in the oxidation of 1-butanol. At 1-butanol concentrations above $4 \mathrm{mM}$, oxidation became incomplete (see equation 3 ). When the substrate concentration reached $8.5 \mathrm{mM}$, butyrate was excreted into the medium in addition to acetate (see equation 4 ).

$$
\begin{aligned}
& \mathrm{CH}_{3} \mathrm{CH}_{2} \mathrm{CH}_{2} \mathrm{CH}_{2} \mathrm{OH}+3 \mathrm{SO}_{4}^{2-} \rightarrow \\
& 4 \mathrm{HCO}_{3}^{-}+3 \mathrm{HS}^{-}+\mathrm{H}^{+}+\mathrm{H}_{2} \mathrm{O} \\
& \mathrm{CH}_{3} \mathrm{CH}_{2} \mathrm{CH}_{2} \mathrm{CH}_{2} \mathrm{OH}+2 \mathrm{SO}_{4}^{2-} \rightarrow \\
& \mathrm{CH}_{3} \mathrm{COO}^{-}+2 \mathrm{HCO}_{3}^{-}+2 \mathrm{HS}^{-}+\mathrm{H}^{+}+\mathrm{H}_{2} \mathrm{O} \\
& \mathrm{CH}_{3} \mathrm{CH}_{2} \mathrm{CH}_{2} \mathrm{CH}_{2} \mathrm{OH}+\mathrm{SO}_{4}^{2-} \rightarrow \\
& 2 \mathrm{CH}_{3} \mathrm{COO}^{-}+\mathrm{HS}^{-}+\mathrm{H}^{+}+\mathrm{H}_{2} \mathrm{O} \\
& \mathrm{CH}_{3} \mathrm{CH}_{2} \mathrm{CH}_{2} \mathrm{CH}_{2} \mathrm{OH}+0 \cdot 5 \mathrm{SO}_{4}^{2-} \rightarrow \\
& \mathrm{CH}_{3} \mathrm{CH}_{2} \mathrm{CH}_{2} \mathrm{CH}_{2} \mathrm{OO}^{-}+0 \cdot 5 \mathrm{HS}^{-}+0 \cdot 5 \mathrm{H}^{+}+\mathrm{H}_{2} \mathrm{O}
\end{aligned}
$$


Table 3. Characteristics of the closest relatives of Desulfotomaculum gibsoniae strain Groll ${ }^{\top}$

Data were taken from Cord-Ruwisch \& Garcia (1985) (D. sapomandens), Daumas et al. (1988) (D. geothermicum), Kuever et al. (1993) (strain Groll ${ }^{\mathrm{T}}$ ) and Fardeau et al. (1995) (D. thermosapovorans). ND, Not determined. Where appropriate, characters are shown as: + , positive; $(+)$, weak; or - , negative.

\begin{tabular}{|c|c|c|c|c|}
\hline Characteristic & $\begin{array}{l}\text { D. gibsoniae } \\
\text { strain Groll }^{\mathrm{T}}\end{array}$ & $\begin{array}{l}\text { D. geothermicum } \\
\text { DSM 3669 }\end{array}$ & $\begin{array}{c}\text { D. thermosapovorans } \\
\text { DSM } 6562^{\mathrm{T}}\end{array}$ & $\begin{array}{l}\text { D. sapomandens } \\
\text { DSM } 3223^{\mathrm{T}}\end{array}$ \\
\hline Cell shape & Rod & Rod & Rod & Rod \\
\hline Cell size $(\mu \mathrm{m})$ & $1 \cdot 5-2 \cdot 5 \times 4-7$ & $0.5-0.8 \times 2.3$ & $1 \cdot 5-2 \cdot 0 \times 5-8$ & $1 \cdot 2-2.0 \times 5-7$ \\
\hline Motility & $+^{*}$ & + & + & + \\
\hline $\mathrm{G}+\mathrm{C}$ content $(\mathrm{mol} \%)$ & $54 \cdot 8$ & $50 \cdot 4$ & $51 \cdot 2$ & 48 \\
\hline Spore shape & Spherical & Spherical & Spherical & Spherical \\
\hline Gas vacuoles & - & + & - & + \\
\hline Optimal growth temperature $\left({ }^{\circ} \mathrm{C}\right)$ & $35-37$ & 54 & 50 & 38 \\
\hline $\mathrm{NaCl}$ requirement & - & $-\dagger$ & - & - \\
\hline Growth factors required & None & None & Vitamins & Vitamins \\
\hline Oxidation of organic substrates & Complete & Complete & Complete & Complete \\
\hline \multicolumn{5}{|l|}{ Electron donors: } \\
\hline $\mathrm{H}_{2} / \mathrm{CO}_{2}$ & + & + & + & - \\
\hline Formate & + & + & + & $(+)$ \\
\hline Acetate & - & - & - & + \\
\hline Propionate & + & + & - & - \\
\hline Butyrate & + & + & + & + \\
\hline C12-C18 fatty acids & - & + & + & + \\
\hline Methanol & - & - & + & ND \\
\hline Ethanol & + & + & + & + \\
\hline Fumarate & + & - & + & $(+)$ \\
\hline Malate & + & ND & + & $(+)$ \\
\hline Benzoate & + & - & - & + \\
\hline Hydroxylated benzoates & + & - & - & + \\
\hline Phenol & + & ND & ND & - \\
\hline Catechol & + & ND & ND & - \\
\hline Methoxy groups of aromatic compounds & + & ND & ND & - \\
\hline Pyruvate & + & ND & + & + \\
\hline Lactate & - & + & + & - \\
\hline Fructose & - & + & - & - \\
\hline \multicolumn{5}{|l|}{ Electron acceptors: } \\
\hline Sulfate & + & + & + & + \\
\hline Thiosulfate & + & ND & + & + \\
\hline Sulfite & + & + & + & + \\
\hline Elemental sulfur & - & - & - & + \\
\hline Nitrate & - & - & - & - \\
\hline Fermentative growth with pyruvate & + & ND & + & - \\
\hline Homoacetogenic growth without sulfate & + & ND & ND & ND \\
\hline
\end{tabular}

* Motility was lost during cultivation.

$\dagger \mathrm{NaCl}$ is not essential; optimal growth in medium with $15 \mathrm{~g} \mathrm{NaCl}^{-\mathbf{1}}$.

$\ddagger$ High substrate concentrations can lead to acetate excretion into the medium.

Spore-forming, sulfate-reducing bacteria like the one described in this paper might be important for the breakdown of organic material in freshwater habitats under varying redox conditions. They can use a large variety of organic compounds, including several aromatic compounds, and can grow even under sulfatefree conditions by homoacetogenesis.
The 16S rRNA gene sequence analyses presented previously (Stackebrandt et al., 1997) and in this study demonstrate that strain Groll $^{\mathrm{T}}$ is a member of the genus Desulfotomaculum and represents a distinct lineage not closely related to any of the previously described species, all of which have been included in the analyses described here. The results of the cellular 
fatty acid composition (Table 2 ) and the physiological properties (Table 3 ), in combination with the results of the 16S rRNA gene sequence comparison, demonstrate that strain Groll $^{\mathrm{T}}$ represents a novel species belonging to phylogenetic subcluster $\mathrm{Ib}$ of the genus Desulfotomaculum. Both of the thermophilic species of subcluster Ib, namely Desulfotomaculum geothermicum and Desulfotomaculum thermosapovorans, share relatively low $(<95 \%)$ 16S rRNA gene sequence similarity with strain Groll ${ }^{\mathrm{T}}$, have much higher optimal growth temperatures and are not able to grow using aromatic compounds as sole carbon sources. Strain Groll ${ }^{\mathrm{T}}$ differs from Desulfotomaculum sapomandens in its ability to grow autotrophically and to grow on a large variety of aromatic compounds. On the basis of the distinct phylogenetic position of strain $\mathrm{Groll}^{\mathrm{T}}$, the demonstrated lack of $16 \mathrm{~S}$ rRNA gene sequence similarity with all previously described Desulfotomaculum species and the differences in physiological and chemotaxonomic characteristics when compared with its closest relatives, we propose the name Desulfotomaculum gibsoniae sp. nov. for strain Groll ${ }^{\mathrm{T}}$.

\section{Description of Desulfotomaculum gibsoniae sp. nov.}

Desulfotomaculum gibsoniae (gib.so'ni.ae M.L. gen. n. gibsoniae of Gibson; named after Jane Gibson, a British-American microbiologist and biochemist, who made important contributions to the field of anaerobic degradation of aromatic compounds).

Straight or slightly curved rods, $1 \cdot 5-2 \cdot 5 \times 4-7 \mu \mathrm{m}$, with pointed ends. Spores spherical and central, causing swelling. No gas vesicles are observed. Cells are motile, but motility was lost during cultivation. Gram-stain reaction of cells in the exponential phase of growth is negative. Strict anaerobe. Growth on $\mathrm{H}_{2} / \mathrm{CO}_{2}$, formate, propionate, butyrate, valerate, capronate, caprylate, iso-butyrate, 2-methylbutyrate, 3-methylbutyrate, crotonate, ethanol, 1-propanol, 1-butanol, hexanol, octanol, 2-methyl-1-propanol, 1,3-propanediol, pyruvate, malate, fumarate, succinate, maleinate, benzoate, 3- and 4-hydroxybenzoate, 2aminobenzoate, phenol, catechol, 3,4-dihydroxybenzoate, phenylacetate, phenylpropionate, aromatic alcohols and aldehydes and methoxylated aromatic compounds. Substrate oxidation is usually complete, leading to $\mathrm{CO}_{2}$, but acetate or other fatty acids can accumulate at high substrate concentrations. Electron acceptors used are sulfate, sulfite and thiosulfate. Nitrate and sulfur are not used. Slow homoacetogenic growth on some compounds. Addition of $\mathrm{NaCl}$ is not necessary. $\mathrm{NaCl}$ is tolerated up to $35 \mathrm{~g} \mathrm{l}^{-1}$. Vitamins are not required for growth. Temperature requirements: $\mathrm{T}_{\text {min }}, 20^{\circ} \mathrm{C} ; \mathrm{T}_{\text {opt }}, 35-37{ }^{\circ} \mathrm{C} ; \mathrm{T}_{\max }, 42{ }^{\circ} \mathrm{C}$. The $\mathrm{pH}$ range for growth is $6-8 ; \mathrm{pH}$ optimum at $6 \cdot 9-7 \cdot 2$. The $\mathrm{G}+\mathrm{C}$ content of the DNA is $54.8 \mathrm{~mol} \%$. The EMBL accession number for the 16S rRNA gene sequence is Y11576. Phylogenetically a member of subcluster Ib of the genus Desulfotomaculum. The type strain of Desulfotomaculum gibsoniae is Groll ${ }^{\mathrm{T}}$ $\left(=\right.$ DSM $\left.7213^{\mathrm{T}}\right)$.

\section{ACKNOWLEDGEMENTS}

The authors thank K.-H. Blotevogel and H. G. Trüper for advice and help. This project was in part supported by the Max-Planck-Society.

\section{REFERENCES}

Campbell, L. L. \& Singleton, R., Jr (1986). Genus IV. Desulfotomaculum Campbell and Postgate 1965, 361 AL. In Bergey's Manual of Systematic Bacteriology, vol. 2, pp. 1200-1202. Edited by P. H. A. Sneath, N. S. Mair, M. E. Sharpe \& J. G. Holt. Baltimore: Williams \& Wilkins.

Cord-Ruwisch, R. \& Garcia, J. L. (1985). Isolation and characterization of an anaerobic benzoate-degrading spore-forming sulfate-reducing bacterium Desulfotomaculum sapomandens $\mathrm{sp}$. nov. FEMS Microbiol Lett 29, 325-330.

Daumas, S., Cord-Ruwisch, R. \& Garcia, J. L. (1988). Desulfotomaculum geothermicum sp. nov., a thermophilic, fatty aciddegrading, sulfate-reducing bacterium isolated with $\mathrm{H}_{2}$ from geothermal ground water. Antonie Leeuwenhoek 54, 165-178.

Fardeau, M.-L., Ollivier, B., Patel, B. K. C., Dwivedi, P., Ragot, M. \& Garcia, J.-L. (1995). Isolation and characterization of a thermophilic sulfate-reducing bacterium, Desulfotomaculum thermosapovorans sp. nov. Int J Syst Bacteriol 45, 218-221.

Felsenstein, J. (1993). PHYLIP (Phylogenetic Inference Package) version 3.5.1. Department of Genetics, University of Washington, Seattle, USA.

Hagenauer, A., Hippe, H. \& Rainey, F. (1997). Desulfotomaculum aeronauticum sp. nov., a spore-forming thiosulfate-reducing bacterium from corroded aluminum alloy in an aircraft. Syst Appl Microbiol 20, 65-71.

Hanselmann, K. W., Kaiser, J. P., Wenk, M., Schön, R. \& Bachofen, R. (1995). Growth on methanol and conversion of methoxylated aromatic substrates by Desulfotomaculum orientis in the presence and absence of sulfate. Microbiol Res 150, 387-401.

Hippe, H., Hagenauer, H. \& Kroppenstedt, R. M. (1997). Menadione requirement for sulfate-reduction in Desulfotomaculum aeronauticum, and emended species description. Syst Appl Microbiol 20, 554-558.

Klemps, R., Cypionka, H., Widdel, F. \& Pfennig, N. (1985). Growth with hydrogen, and further physiological characteristics of Desulfotomaculum sp. Arch Microbiol 143, 203-208.

Kuever, J., Kulmer, J., Jannsen, S., Fischer, U. \& Blotevogel, K.-H. (1993). Isolation and characterization of a new spore-forming sulfate-reducing bacterium growing by complete oxidation of catechol. Arch Microbiol 159, 282-288.

Liu, Y., Karnauchow, T. M., Jarrell, K. F., Balkwill, D. L., Drake, G. R., Ringelberg, D., Clarno, R. \& Boone, D. R. (1997). Description of two new thermophilic Desulfotomaculum spp., Desulfotomaculum putei sp. nov., from a deep terrestrial subsurface, and Desulfotomaculum luciae sp. nov., from a hot spring. Int J Syst Bacteriol 47, 615-621.

Londrey, K. L., Fedorak, P. M. \& Suflita, J. M. (1997). Anaerobic degradation of $\mathrm{m}$-cresol by a sulfate-reducing bacterium. Appl Environ Microbiol 63, 3170-3175.

Love, C. A., Patel, B. K. C., Nichols, P. D. \& Stackebrandt, E. (1993). Desulfotomaculum australicum $\mathrm{sp}$. nov., a thermophilic sulfatereducing bacterium isolated from the Great Artesian Basin of Australia. Syst Appl Microbiol 16, 244-251.

Maidak, B. L., Olsen, G. J., Larsen, N., Overbeek, R., McCaughey, M. J. \& Woese, C. R. (1996). The Ribosomal Database Project (RDP). Nucleic Acids Res 24, 82-85. 
Min, H. \& Zinder, S. H. (1990). Isolation and characterization of a thermophilic sulfate-reducing bacterium Desulfotomaculum thermoacetoxidans sp. nov. Arch Microbiol 153, 399-404.

Nazina, T. N., Ivanova, A. E., Kanchaveli, L. P. \& Rozanova, E. P. (1989). A new spore-forming thermophilic methylotrophic bacterium, Desulfotomaculum kuznetsovii sp. nov. Microbiology (English translation of Mikrobiologiya) 57, 659-663.

Newman, D. K., Kennedy, E. K., Coates, J. D., Ahmann, D., Ellis, D. J., Lovley, D. R. \& Morel, F. M. M. (1997). Dissimilatory arsenate and sulfate reduction in Desulfotomaculum auripigmentum $\mathrm{sp}$. nov. Arch Microbiol 168, 380-388.

Nilsen, R. K., Torsvik, T. \& Lien, T. (1996). Desulfotomaculum thermocisternum sp. nov., a sulfate reducer isolated from a hot North Sea oil reservoir. Int $J$ Syst Bacteriol 46, 397-402.

Postgate, J. R. (1956). Cytochrome $c_{3}$ and desulphoviridin; pigments of the anaerobe Desulphovibrio desulphuricans. J Gen Microbiol 14, 545-572.

Schauder, R., Eikmanns, B., Thauer, R. K., Widdel, F. \& Fuchs, G. (1986). Acetate oxidation to $\mathrm{CO}_{2}$ in anaerobic bacteria via a novel pathway not involving reactions of the citric acid cycle. Arch Microbiol 145, 162-172.

Stackebrandt, E., Sproer, C., Rainey, F. A., Burghardt, J., Păuker, O. \& Hippe, H. (1997). Phylogenetic analysis of the genus Desulfotomaculum: evidence for the misclassification of $D e$ sulfotomaculum guttoideum and description of Desulfotomaculum orientis as Desulfosporosinus orientis gen. nov., comb. nov. Int $J$ Syst Bacteriol 47, 1134-1139.

Tardy-Jacquenod, C., Magot, M., Patel, B. K. C., Matheron, R. \&
Caumette, P. (1998). Desulfotomaculum halophilum sp. nov., a halophilic sulfate-reducing bacterium isolated from oil production facilities. Int $J$ Syst Bacteriol 48, 333-338.

Tasaki, M., Kamagata, Y., Nakamura, K. \& Mikami, E. (1991). Isolation and characterization of a thermophilic benzoatedegrading, sulfate-reducing bacterium, Desulfotomaculum thermobenzoicum sp. nov. Arch Microbiol 155, 348-352.

Tasaki, M., Kamagata, Y., Nakamura, K. \& Mikami, E. (1992). Utilization of methoxylated benzoates and formation of intermediates by Desulfotomaculum thermobenzoicum in the presence or absence of sulfate. Arch Microbiol 157, 209-212.

Vainshtein, M. B., Hippe, H. \& Kroppenstedt, R. M. (1992). Cellular fatty acid composition of Desulfovibrio species and its use in classification of sulfate-reducing bacteria. Syst Appl Microbiol 15, 554-566.

Vainshtein, M. B., Gogotova, I. G. \& Hippe, H. (1995). A sulfatereducing bacterium from permafrost. Microbiology (English translation of Mikrobiologiya) 64, 436-439.

Widdel, F. (1992). The Genus Desulfotomaculum. In The Prokaryotes, 2nd edn, pp. 1792-1799. Edited by A. Balows, H. G. Trüper, M. Dworkin, W. Harder \& K. H. Schleifer. New York: Springer.

Widdel, F. \& Pfennig, N. (1977). A new anaerobic, sporing, acetate-oxidizing, sulfate-reducing bacterium, Desulfotomaculum (emend.) acetoxidans. Arch Microbiol 112, 119-122.

Widdel, F. \& Pfennig, N. (1981). Sporulation and further nutritional characteristics of Desulfotomaculum acetoxidans. Arch Microbiol 129, 401-402. 